\title{
On the superheavy elements formed in capture of very heavy ions at high excitation energies
}

\author{
G. Royer \\ Laboratoire Subatech, UMR: IN2P3/CNRS-Université-Ecole des Mines, Nantes 44, France
}

\begin{abstract}
The potential barriers governing the reactions ${ }^{58} \mathrm{Fe}+{ }^{244} \mathrm{Pu},{ }^{238} \mathrm{U}+{ }^{64} \mathrm{Ni}$ and ${ }^{238} \mathrm{U}+{ }^{72} \mathrm{Ge}$ have been determined from a liquid drop model taking into account the proximity energy, the shell energies, the rotational energy, and the deformation of the incoming nuclei in the quasimolecular shape valley. Double-humped potential barriers appear in these entrance channels. The external saddle-point corresponds to two touching ellipsoidal nuclei when the shell and pairing effects are taken into account while the inner barrier is due to the shell effects at the vicinity of the spherical shape of the composite system. Between them, a large potential pocket exists and persists at very high angular momenta allowing the capture of very heavy ions at high excitation energies.
\end{abstract}

PACS numbers: 24.75.+i,25.70.J,25.70.-Z,27.90.+b

Using cold fusion reactions of mean mass asymmetry involving a doubly magic spherical ${ }^{208} \mathrm{~Pb}$ or a close $\mathrm{Bi}$ target and $\mathrm{Cr}, \mathrm{Fe}, \mathrm{Ni}$ and $\mathrm{Zn}$ deformed projectiles and, more recently, highly asymmetric warm fusion reactions using a doubly magic spherical projectile ${ }^{48} \mathrm{Ca}$ and $\mathrm{Np}$, $\mathrm{Pu}, \mathrm{Am}, \mathrm{Cm}, \mathrm{Bk}$ and $\mathrm{Cf}$ targets, new very heavy elements have been synthesized [1-6]. In these experiments, the purpose was always to minimize the excitation energy of the composite system to reduce the neutron emission and to try to stabilize the nucleon shells. The selected beam energy corresponds roughly to the fusion barrier height and, from the compound nuclei, the fusion evaporation residues emerge after cooling by neutron emission and are detected by their $\alpha$ decay chains ending by fission. So the half-lives of these heaviest isotopes at the northeast of the table of isotopes actually merge into their $\alpha$ decay half-lives and can reach several seconds.

Recently, the systems ${ }^{208} \mathrm{~Pb}+\mathrm{Ge}, \quad{ }^{238} \mathrm{U}+\mathrm{Ni}$ and ${ }^{238} \mathrm{U}+\mathrm{Ge}$ have been studied at high excitation energy of $6.16,6.62$ and $6.09 \mathrm{MeV} / \mathrm{u}$ possibly leading to nuclear systems of charge 114, 120 and 124 respectively $[7,8]$. Reverse kinematics allows the measurement of the fragment atomic number and kinetic energy. Capture reactions in which all the projectile and target protons form a composite system that will dominantly split in two heavy fissionlike fragments have been identified with a $4 \pi$ charged product detection array. An analysis of the nuclear reaction time distributions measured by the blocking technique in single crystals and, later on, based on x-ray fluorescence yields provides evidence for composite systems with $\mathrm{Z}=120$ and 124 living longer than $10^{-18} \mathrm{~s}$. This fission time being at least 2 orders of magnitude longer than the usually measured QuasiFission characteristic times and that the longest lifetimes of giant composite systems calculated in transuranium ion collisions, it has been concluded that these detected events are fusion-fission events from highly excited compound nuclei. By contrast, the neutron deficient nucleus of charge 114 possibly formed in the ${ }^{208} \mathrm{~Pb}+\mathrm{Ge}$ reaction have lifetimes close to or below the sensitivity limit of the experiment [7].
The purpose of the present study is to determine the L-dependent potential barriers governing these reactions within a generalized liquid drop model (GLDM) taking into account the mass and charge asymmetries, the proximity energy, the shell and pairing effects and rotational energies. This macro-microscopic approach has been used to explore the data on the symmetric and asymmetric fission [9, 10], $\alpha$ and light nucleus emission [11], fusion data $[12,13]$ as well as the rotating highly deformed state characteristics [14].

The GLDM energy is the sum of the volume, surface, Coulomb and nuclear proximity energies [9, 12].

All along the fusion path the proximity energy term takes into account the nuclear attractive forces between nucleons across the gap between the incoming close separated nuclei and, later on, in the neck of the deformed one-body system. For example, at the contact point between two spherical $\mathrm{U}$ and $\mathrm{Ni}$ nuclei the proximity energy reaches $-44 \mathrm{MeV}$. The absence of this term in fusion studies leads to an unrealistic Coulomb peak.

In the entrance channels where the necks are narrow and well developed this correction to the surface energy plays an important role on a large part of the potential barrier and specially around the contact point.

The shape-dependent shell corrections have been determined within the Droplet Model formulas $[9,15]$ and the pairing energy has been calculated within the Thomas-Fermi model [9].

The selected shape sequence simulating the fusion valley is shown in Fig. 1. The coaxial ellipsoidal deformations of the projectile and target are considered and the nuclei may have independently oblate or prolate deformations. The rapid development of a deep neck while keeping almost spherical ends is described using two elliptic lemniscatoids joined in a plan perpendicular to the fusion axis [12]. The proximity energy is maximized in this deformation path.

Firstly, the kinetic energy in the center of mass, the excitation energy and the $\mathrm{Q}$ value are given in Table I for the reactions described in Ref. [7, 8] and also, for comparison, for the reaction ${ }^{58} \mathrm{Fe}+{ }^{244} \mathrm{Pu}$ studied previ- 


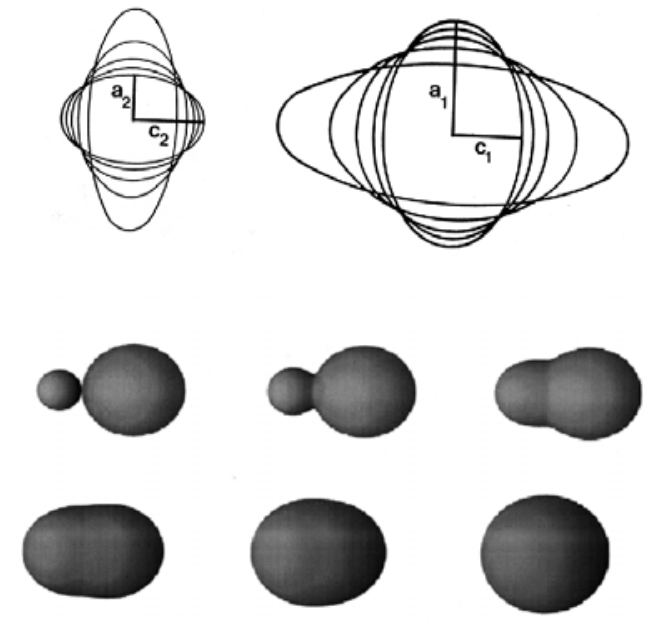

FIG. 1: Shape sequences describing the entrance channel : two separated ellipsoidal incoming nuclei and one-body quasimolecular shape for a given initial asymmetry.

ously $[16,17]$. The excitation energy is very high for the two first reactions and lower for the ${ }^{58} \mathrm{Fe}+{ }^{244} \mathrm{Pu}$ reaction.

\begin{tabular}{|c|c|c|c|}
\hline Reaction & Kinetic energy & Excitation Energy & $\mathrm{Q}$ \\
\hline${ }_{92}^{238} \mathrm{U}+{ }_{28}^{64} \mathrm{Ni} \rightarrow{ }_{120}^{302} \mathrm{Sh}$ & 333.9 & 95 & 238.9 \\
${ }_{92}^{238} \mathrm{U}+{ }_{32}^{72} \mathrm{Ge} \rightarrow{ }_{124}^{310} \mathrm{Sh}$ & 336.6 & 55.6 & 281.0 \\
${ }_{26}^{58} \mathrm{Fe}+{ }_{94}^{244} \mathrm{Pu} \rightarrow{ }_{120}^{302} \mathrm{Sh}$ & 259.8 & 38.35 & 221.45 \\
\hline
\end{tabular}

TABLE I: Kinetic energy, excitation energy and reaction $Q$ value (in $\mathrm{MeV}$ ) for the three indicated reactions $[7,16,17]$.

The dependence of the inner barrier close to the sphere on the next assumed magic proton number is displayed in Fig. 2 for the ${ }_{120}^{302} \mathrm{Sh}$ nucleus. The fission barrier height can reach $12-14 \mathrm{MeV}$ if the next proton magic number is 120 .

The L-dependent capture barriers determined in this quasimolecular shape valley are shown in Figs [3-5]. The solid line indicates for $L=0$ the potential energy calculated in taking into account the ellipsoidal deformations of the incoming nuclei and their shell and pairing energies in the upper part (a). The other curves display for two-body shapes the macroscopic energy of two separated spheres. For each reaction the barriers displayed in the upper part of the figure include the shell and pairing effects around the sphere of the composite system and, to maximize these shell effects, the assumed next magic proton number is the proton number of the compound nucleus. In the lower part (b), the shell effects around the sphere and for two spheres or two ellipsoids at $L=0$ are disregarded. It has been previously shown that such barriers allow a good reproduction of the empirical fusion barrier heights and positions $[12,13]$. In Table II the GLDM fusion barrier heights are compared with the ones derived from the Bass empirical potential [18]. The

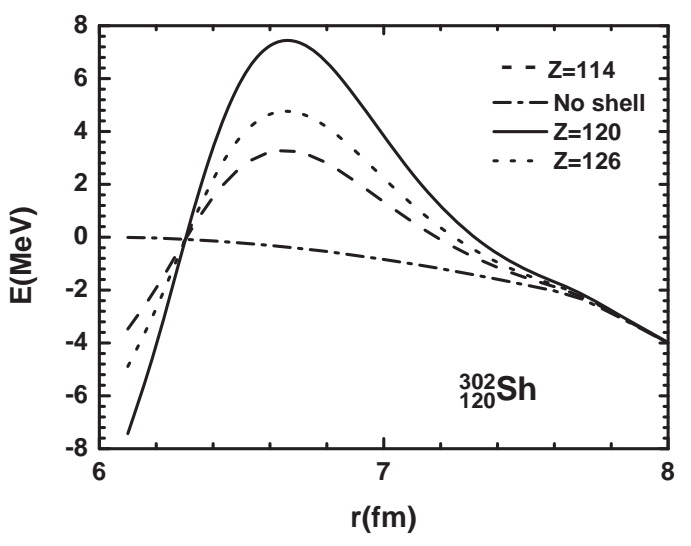

FIG. 2: Fission barrier of the ${ }_{120}^{302} \mathrm{Sh}$ nucleus as a function of the assumed next magic proton number. $r$ is the distance between the mass centers.

values are very close to each other whatever the asymmetry and the mass of the system are. The fusion barrier radii are also very similar ([18] p 336, last column), the difference between the two radii being at most $0.1 \mathrm{fm}$.

Macroscopically, as for all fusion reactions, an external fusion barrier appears and corresponds to an unstable equilibrium due to the balance between the repulsive Coulomb forces and the attractive nuclear proximity forces between separated incoming nuclei. After the neck formation the proximity energy introduces an inflexion in the potential curve which flattens the potential surface independently of the shell effects which generate the inner peak close to the sphere. Between the two peaks of these double-humped capture barriers there is an energy degeneracy on a range of about 3 fms. Quasimolecular isomeric states can survive a long time in this large potential pocket since the neck between the two nuclei is formed and exchanges of nucleons can occur, the external barrier being sufficiently high to prevent a rapid decay.

In these fusion reactions the deformations of the projectile or target and their shell and pairing energies may play some role and calculations have been done at $L=0$. Extrapolations including an angular momentum may be performed easily. The transition between onebody and two-body shapes is less smooth when the ellipsoidal deformations of the nuclei are taken into account. All the macro-microscopic models and the Hartree-FockBogoliubov approaches have difficulties smoothly connecting these two sheets of the potential energy surface. Then the external maximum of the barrier corresponds to the transition from two touching deformed nuclei to a one-body quasi-molecular shape with a very deep neck. The height and the radius of the fusion barriers are reduced. More precisely and for the $\mathrm{U}+\mathrm{Ni}$ reaction the barrier top lies at $\mathrm{r}=10.8 \mathrm{fm}$ while the contact point between two spherical nuclei corresponds to $\mathrm{r}=12 \mathrm{fm}$. The two incoming nuclei are oblate at the saddle-point with 
TABLE II: Comparison between the GLDM and the Bass fusion barrier heights (in MeV).

\begin{tabular}{cccccccccccc}
\hline \hline Reaction & $B_{G L D M}$ & $B_{\text {Bass }}$ & Reaction & $B_{\text {GLDM }}$ & $B_{\text {Bass }}$ & Reaction & $B_{\text {GLDM }}$ & $B_{\text {Bass }}$ & Reaction & $B_{\text {GLDM }}$ & $B_{\text {Bass }}$ \\
\hline${ }^{12} \mathrm{C}+{ }^{14} \mathrm{~N}$ & 6.85 & 7.03 & ${ }^{16} \mathrm{O}+{ }^{27} \mathrm{Al}$ & 15.8 & 16.0 & ${ }^{32} \mathrm{~S}+{ }^{24} \mathrm{Mg}$ & 28.0 & 28.2 & ${ }^{18} \mathrm{O}+{ }^{58} \mathrm{Ni}$ & 31.2 & 31.2 \\
${ }^{12} \mathrm{C}+{ }^{152} \mathrm{Sm}$ & 46.6 & 46.8 & ${ }^{32} \mathrm{~S}+{ }^{58} \mathrm{Ni}$ & 60.0 & 60.1 & ${ }^{35} \mathrm{Cl}+{ }^{90} \mathrm{Zr}$ & 85.2 & 85.3 & ${ }^{40} \mathrm{Ar}+{ }^{110} \mathrm{Pd}$ & 99.2 & 99.3 \\
${ }^{84} \mathrm{Kr}+{ }^{72} \mathrm{Ge}$ & 134.6 & 135.1 & ${ }^{40} \mathrm{Ar}+{ }^{197} \mathrm{Au}$ & 157.0 & 157.3 & ${ }^{84} \mathrm{Kr}+{ }^{116} \mathrm{Cd}$ & 190.6 & 191.6 & ${ }^{48} \mathrm{Ca}+{ }^{248} \mathrm{Cm}$ & 200.8 & 201.1 \\
${ }^{52} \mathrm{Cr}+{ }^{208} \mathrm{~Pb}$ & 211.1 & 211.9 & ${ }^{54} \mathrm{Cr}+{ }^{244} \mathrm{Pu}$ & 235.0 & 235.8 & ${ }^{64} \mathrm{Ni}+{ }^{238} \mathrm{U}$ & 265.4 & 266.4 & ${ }^{64} \mathrm{Ni}+{ }^{248} \mathrm{Cm}$ & 275.4 & 276.5 \\
${ }^{70} \mathrm{Zn}+{ }^{238} \mathrm{U}$ & 281.9 & 283.0 & ${ }^{68} \mathrm{Zn}+{ }^{238} \mathrm{U}$ & 283.1 & 284.3 & ${ }^{70} \mathrm{Zn}+{ }^{244} \mathrm{Pu}$ & 287.0 & 288.1 & $74 \mathrm{Ge}+{ }^{232} \mathrm{Th}$ & 294.1 & 295.3 \\
${ }^{74} \mathrm{Ge}+{ }^{238} \mathrm{U}$ & 299.7 & 300.7 & ${ }^{80} \mathrm{Se}+{ }^{232} \mathrm{Th}$ & 310.2 & 311.3 & ${ }^{86} \mathrm{Kr}+{ }^{236} \mathrm{Ra}$ & 320.0 & 321.1 & ${ }^{130} \mathrm{Te}+{ }^{176} \mathrm{Yb}$ & 365.9 & 365.5 \\
\hline \hline
\end{tabular}
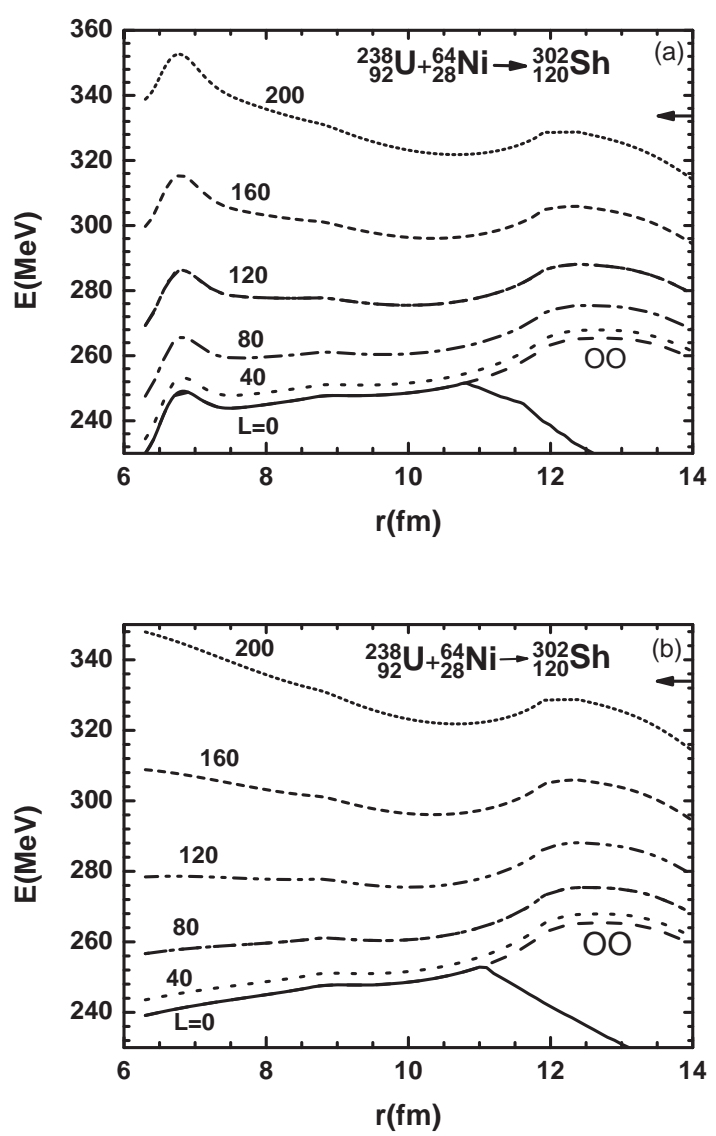

FIG. 3: Potential barrier for the ${ }_{92}^{238} \mathrm{U}+{ }_{28}^{64} \mathrm{Ni} \rightarrow{ }_{120}^{302} \mathrm{Sh}$ reaction versus the angular momentum ( $\hbar$ unit), including the shell and pairing effects for the one-body shape in the upper part (a) and not in the lower part (b). The arrow indicates the beam energy. The solid line at $L=0$ takes into account the ellipsoidal deformations of the target and projectile and their shell and pairing energies in the upper part of the figure and not in the lower part.

a ratio $s_{i}=a_{i} / c_{i}$ (see Fig. 1 ) between the semi-axes of $s_{1}=1.18$ (U nucleus) and $s_{2}=1.16$ (Ni nucleus) respectively. The evolution of the shapes of the two nuclei is governed by the balance between the variations of the shell, proximity and Coulomb effects. At $11.7 \mathrm{fm}$ there is a shape transition : $s_{1}=1.16-0.86$ and $s_{2}=0.92-1.5$. At
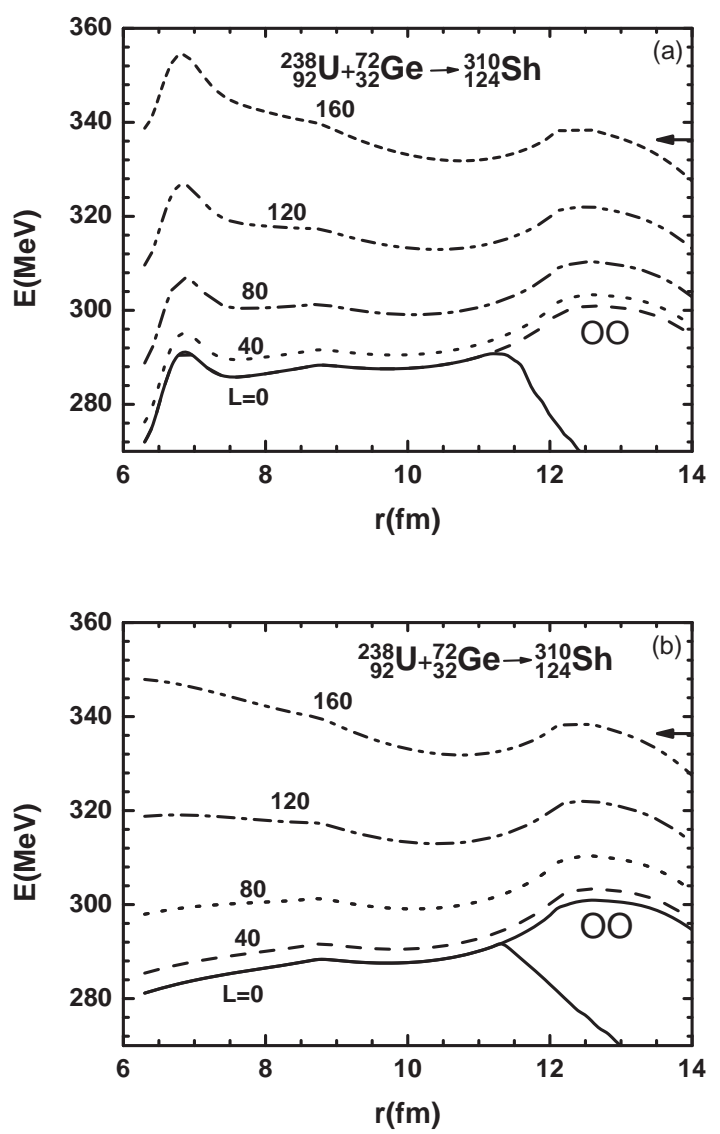

FIG. 4: Same as Fig. 3 but for the ${ }_{92}^{238} \mathrm{U}+{ }_{32}^{72} \mathrm{Ge} \rightarrow{ }_{124}^{310} \mathrm{Sh}$ reaction.

$\mathrm{r}=15.5 \mathrm{fm}$ the proximity forces begin to play a role in the entrance channel and induce a transition from an oblate shape to a prolate one for the heaviest nucleus, $s_{1}$ varying from 1.22 to 0.56 . It corresponds to the sticking of the two nuclei and to the scission point in the exit channel [9]. When the microscopic effects are not taken into account the transition between two-body and one-body shapes occurs at $\mathrm{r}=11 \mathrm{fm}$ and $s_{1}=1.04$ ( $\mathrm{U}$ nucleus) and $s_{2}=1.34$. The considered deformations are coaxial but the two nuclei may evolve independently. The introduction of an orientation between the axes of the ellipsoids still would diminish slightly the fusion barrier height and 

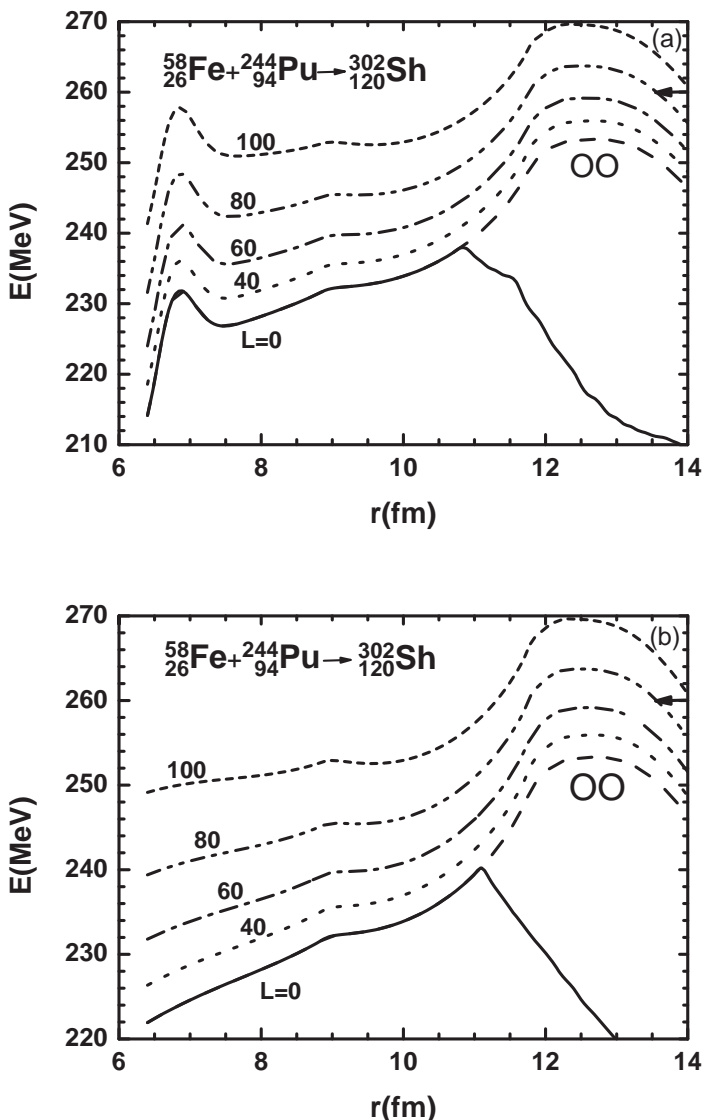

FIG. 5: Same as Fig. 3 but for the ${ }_{26}^{58} \mathrm{Fe}+{ }_{94}^{244} \mathrm{Pu} \rightarrow{ }_{120}^{302} \mathrm{Sh}$ reaction.

radius. Then the possibility of a rapid decay increases at low angular momentum but the macroscopic picture remains at very high angular momenta since the shell effects progressively disappear with increasing angular momenta and are probably partially destroyed at the highest angular momenta.

In the ${ }^{238} \mathrm{U}+\mathrm{Ni}$ and ${ }^{238} \mathrm{U}+\mathrm{Ge}$ reactions the excitation energy of the composite system is very large and allows to populate highly rotating states of more than $160 \hbar$, the excitation energy being much smaller in the ${ }^{58} \mathrm{Fe}+\mathrm{Pu}$ reaction. So, the possibility exists of the formation and stability of rapidly rotating isomeric states without necessarily reaching a quasi-spherical nuclear shape and even though the shell effects vanish and the inner barrier is destroyed. Incomplete fusion and quasifission events from the external pocket are the main exit channels for these isomeric rotational states while evaporation residues living possibly several seconds come from a compound nucleus.

If the reorganization of the single particle levels is very rapid then the value of the proton magic number begins to play some role to reach the configuration of a quasispherical compound nucleus. So an open question is whether at large deformations and high angular momenta the nucleons shells can take form to stabilize the nuclear system before investigating a peculiar exit channel. The pre or post equilibrium nature of the neutron evaporation process is also crucial.

It has been shown that for these reactions between very heavy ions appear a dynamic hindrance against fusion since the two incoming nuclei are very close to each other at the top of the external fusion barrier and energy dissipation occurs due to friction forces $[12,19]$. The product of charges $Z_{1} Z_{2}$ plays a main role, it reaches 2460 and 2490 respectively for the heaviest presently successful cold reactions $\mathrm{Zn}+\mathrm{Pb}$ and $\mathrm{Zn}+\mathrm{Bi}$ which, also, optimize the Q-Value. For the heaviest successful warm reaction $\mathrm{Ca}+\mathrm{Cf}, \mathrm{Z}_{1} \mathrm{Z}_{2}$ is only 1960 much below the limit which seems to be around 2400-2500. So the reaction $\mathrm{Fe}+\mathrm{Pu}$ $\left(\mathrm{Z}_{1} \mathrm{Z}_{2}=2444\right)$ is at the limit of appearance of this supplementary energy needed to pass the barrier and, furthermore, it does not optimize the Q-value. The $\mathrm{U}+\mathrm{Ni}$ and $\mathrm{U}+\mathrm{Ge}$ reactions lead to still higher values of 2576 and 2944.
[1] S. Hofmann et al., Eur. Phys. J. A 32, 251 (2007) and references therein.

[2] A. K. Nasirov et al., Phys. Rev. C 79, 024606 (2009).

[3] S. Hofmann et al., Eur. Phys. J. A 48, 62 (2012).

[4] Yu. Ts. Oganessian et al., Phys. Rev. C 74, 044602 (2006) and references therein.

[5] Yu. Ts. Oganessian et al., Phys. Rev. Lett. 108, 022502 (2012) and references therein.

[6] K. Morita et al., J. Phys. Soc. Jpn. 76, 045001 (2007); J. Phys. Soc. Jpn. 81, 103201 (2012).

[7] M. Morjean et al., Phys. Rev. Lett. 101, 072701 (2008).

[8] M. O. Frégeau et al., Phys. Rev. Lett. 108, 122701 (2012).

[9] G. Royer, M. Jaffré, and D. Moreau, Phys. Rev. C 86, 044326 (2012).
[10] X. J. Bao, H. F. Zhang, G. Royer, and J. Q. Li, Nucl. Phys. A 906, 1 (2013).

[11] G. Royer and R. Moustabchir, Nucl. Phys. A 683, 182 (2001).

[12] G. Royer and B. Remaud, Nucl. Phys. A 444, 477 (1985).

[13] G. Royer and J. Gaudillot, Phys. Rev. C 84, 044602 (2011).

[14] G. Royer, C. Bonilla, and R. A. Gherghescu, Phys. Rev. C 67, 034315 (2003).

[15] W.D. Myers, Droplet model of atomic nuclei (Plenum, New-York, 1977).

[16] N. Amar, Thesis Caen (2003), unpublished.

[17] I. Itkis et al., Proceedings of the international conference : Nuclear physics at Border Lines, edited by G. Fazio et al. (World Scientific, Singapore, 2002), p. 142. 
[18] R. Bass, Nuclear reactions with heavy ions (Springer, [19] W. J. Swiatecki, Nucl. Phys. A 376, 275 (1982). Berlin, 1980) p 336 and 345 . 